\title{
On the supply of heavy planetary material to the magnetotail of Mercury
}

\author{
D. C. Delcourt \\ LPP, Ecole Polytechnique-CNRS-UPMC, Saint-Maur des Fossés, France \\ Correspondence to: D. C. Delcourt (dominique.delcourt@1pp.polytechnique.fr) \\ Received: 18 July 2013 - Accepted: 2 September 2013 - Published: 8 October 2013
}

\begin{abstract}
We examine the transport of low-energy heavy ions of planetary origin $\left(\mathrm{O}^{+}, \mathrm{Na}^{+}, \mathrm{Ca}^{+}\right)$in the magnetosphere of Mercury. We show that, in contrast to Earth, these ions are abruptly energized after ejection into the magnetosphere due to enhanced curvature-related parallel acceleration. Regardless of their mass-to-charge ratio, the parallel speed of these ions is rapidly raised up to $\sim 2 V_{E \times B}$ (denoting by $V_{E \times B}$ the magnitude of the local $\boldsymbol{E} \times \boldsymbol{B}$ drift speed), in a like manner to Fermi-type acceleration by a moving magnetic mirror. This parallel energization is such that ions with very low initial energies (a few tenths of eVs) can overcome gravity and, regardless of species or convection rate, are transported over comparable distances into the nightside magnetosphere. The region of space where these ions reach the magnetotail is found to extend over altitudes similar to those where enhanced densities are noticeable in the MESSENGER data, viz., from $\sim 1000 \mathrm{~km}$ up to $\sim 6000 \mathrm{~km}$ in the pre-midnight sector. The observed density enhancements may thus follow from $\boldsymbol{E} \times \boldsymbol{B}$ related focusing of planetary material of dayside origin into the magnetotail. Due to the planetary magnetic field offset, an asymmetry is found between drift paths anchored in the Northern and Southern hemispheres, which puts forward a predominant role of heavy material originating in the Northern Hemisphere in populating the innermost region of Mercury's magnetotail.
\end{abstract}

Keywords. Magnetospheric Physics (Planetary magnetospheres).

\section{Introduction}

The MESSENGER spacecraft was inserted into orbit around Mercury on 18 March 2011. Since then, in addition to confirming the pioneering Mariner 10 results concerning, e.g., the existence of an intrinsic magnetic field, MESSENGER has revealed a number of new and remarkable features concerning both the planet itself and its neutral and ionized environments. Among these is the discovery of planetary ions in various regions of the Hermean magnetosphere (e.g., Zurbuchen et al., 2008, 2011). In this respect, Raines et al. (2013) recently provided a review of the first years of plasma ion observations from the FIPS instrument on board MESSENGER. In this study, Raines et al. classified heavy ions of planetary origin into two distinct groups, namely an $\mathrm{O}^{+}$-group with mass-to-charge ratio $m / q$ varying from 16 to $20 \mathrm{amu} \mathrm{e}{ }^{-1}$, and an $\mathrm{Na}^{+}$group with $m / q$ between 21 and 30 amu $\mathrm{e}^{-1}$. Although plasma parameters for Mercury cannot be fully characterized because of the limited field of view of the FIPS instrument, the results obtained for the observed density indicate that the $\mathrm{Na}^{+}$group dominates the contribution of planetary ions at Mercury (see, e.g., Table 1 of Raines et al., 2013).

Of particular interest, the statistical analysis of observed density as a function of altitude and longitude in Raines et al. (2013) reveals localized enhancements of the $\mathrm{Na}^{+}$group at equatorial latitudes. These enhancements can be appreciated in Fig. 1, which is adapted from Fig. 3 of Raines et al. (2013). The left panel of Fig. 1 shows two peaks of observed density, the first one near $1000 \mathrm{~km}$ altitude in the dawn sector and the second one extending from $\sim 1000 \mathrm{~km}$ up to $\sim 6000 \mathrm{~km}$ altitude in the pre-midnight sector. Raines et al. (2013) noted that this latter feature appears to be part of a single feature with prominent asymmetry between the 


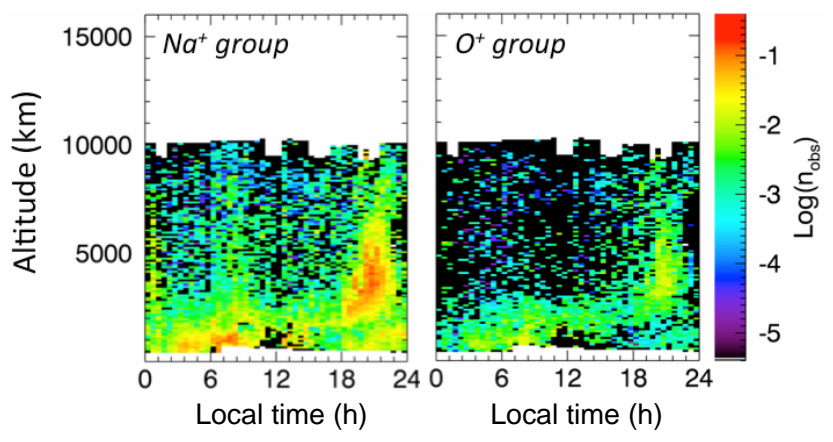

Fig. 1. Observed densities of $\mathrm{Na}^{+}$-group and $\mathrm{O}^{+}$-group ions as measured by the FIPS instrument on board MESSENGER. Densities are shown as a function of local time and altitude, and coded according to the color scale at right (adapted from Raines et al., 2013).

dawn and dusk sectors. These authors also pointed out that the $\mathrm{O}^{+}$group exhibits features similar to those of the $\mathrm{Na}^{+}$ group, but with lower density on average, as evidenced by the right panel of Fig. 1. It was suggested in the study of Raines et al. (2013) that the enhancement visible in the dawn sector results from local desorption of surface material as it enters into the sunlit hemisphere, as put forward in previous modeling studies (e.g., Leblanc and Johnson, 2010). The second enhancement over a larger range of altitudes in the pre-midnight sector is the one of interest in the present study. We will show that it may be related to the escape of heavy planetary material at high latitudes in the northern dayside sector in a very specific manner.

\section{Parallel energization of heavy planetary material}

It was pointed out by Cladis (1986) that, during transport from high to low latitudes, ions expelled from the topside terrestrial ionosphere may experience substantial acceleration in the parallel direction. The approach adopted in Cladis (1986) was based on the guiding center approximation that is not valid for describing the ion motion in the magnetotail field reversal where the magnetic moment (first adiabatic invariant) may not be conserved, but this guiding center approach adequately accounts for ion transport in the magnetospheric lobe where magnetic field variations are negligible within a particle cyclotron turn. In this guiding center (adiabatic) approximation, the parallel equation of motion in the absence of parallel electric can be written as (see, e.g., Eq. 1.20 of Northrop, 1963):

$\dot{V}_{/ /}=g_{\|}+\boldsymbol{V}_{E \times B} \cdot\left[\frac{\partial \boldsymbol{b}}{\partial t}+V_{\|} \frac{\partial \boldsymbol{b}}{\partial s}+\boldsymbol{V}_{E \times B} \cdot \nabla \boldsymbol{b}\right]-\frac{\mu}{m} \frac{\partial B}{\partial s}$.

In this equation, $g_{\|}$is the gravitational acceleration in the parallel direction, $V_{\|}$the particle parallel speed, $\boldsymbol{b}$ a unit vector in the $\boldsymbol{B}$ direction, $s$ the curvilinear abscissa along the field line, and $\mu$ the particle magnetic moment, respectively.
It is important to note here that a similar equation (with the exception of the mirror force term) can be obtained if one considers not the guiding center, but the full equation of motion (see, e.g., Sect. 3.2 of Delcourt et al., 1990). Equation (1) displays two distinct curvature-related acceleration terms, namely, the third and fourth terms on the right hand side of (1) that are due to curvature of the magnetic field lines and curvature of the $\boldsymbol{E} \times \boldsymbol{B}$ drift paths, respectively. Because of the small spatial scales of the Hermean environment, it was shown by Delcourt et al. (2002) that, during convection from dayside to nightside sectors over the polar cap, the curvature-related acceleration in the parallel direction is much more pronounced at Mercury than at Earth, leading to large planetary ion energies (possibly up to several hundreds of eVs or a few keVs) in the lobes and until entry into the plasma sheet. As described below, this curvature-related acceleration may play a prominent role in the pattern observed by MESSENGER for both $\mathrm{Na}^{+}$-group and $\mathrm{O}^{+}$-group densities.

We first note that heavy populations are expelled from the surface of Mercury, with a few tenths of $\mathrm{eV}$ on the average - and at most a few eVs - via different processes (thermal desorption, micro-meteoroid vaporization, photon stimulated desorption, solar wind sputtering). In contrast to hydrogen, these heavy populations thus lie essentially in the immediate vicinity of the planet surface, the scale height of exospheric sodium and oxygen being of the order of a few tens of kilometers (e.g., Hunten et al., 1988). Once ionized by solar UV radiation, the heavy ions produced can participate in the overall plasma circulation within Mercury's environment. Figure 2 shows an example of a test $\mathrm{Na}^{+}$trajectory obtained using a modified version of the Luhmann and Friesen (1979) model with the following parameters: $B_{\mathrm{T}}=70 \mathrm{nT}, L=0.5 \mathrm{R}_{\mathrm{M}}$ and $B_{\mathrm{EQ}}=195 \mathrm{nT}$ ( $B_{\mathrm{T}}$ being the asymptotic tail field, $L$ the half-thickness of the current sheet and $B_{\mathrm{EQ}}$ the equatorial surface field). As revealed by MESSENGER (Anderson et al., 2011), a $484 \mathrm{~km}$ northward offset of the planetary magnetic field was also accounted for in this model. As for the large-scale convection electric field, a twocell Volland-Stern pattern (e.g., Volland, 1978) was considered in first approximation. Also, the full equation of motion was used to compute the ion trajectories in order to account for possible nonadiabatic transport features. This equation was integrated using a fourth-order Runge-Kutta technique.

Figure 2 presents three distinct particle orbits, corresponding to three different cross-polar cap potential drops (denoted as $\Delta \Phi$ hereafter). Note that, in the left panels of Fig. 2 as well as in the following figures, the $x$ axis is oriented from the center of the planet toward the tail, the $y$ axis is oriented from dusk to dawn, and the $z$ axis points northward. For the three cases shown in Fig. 2, the test $\mathrm{Na}^{+}$ions were ejected immediately above the planet surface with an energy of $0.1 \mathrm{eV}$, that is, well below the escape energy (of the order of $2 \mathrm{eV}$ for $\mathrm{Na}^{+}$). For the $\mathrm{Na}^{+}$orbit coded in grey in Fig. 2, an unrealistically low convection electric field was 

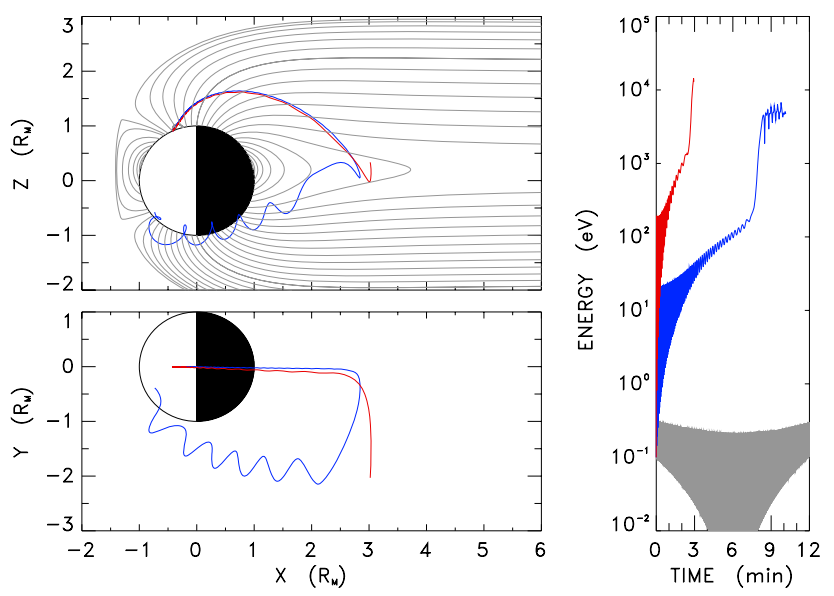

Fig. 2. Model $\mathrm{Na}^{+}$trajectories: (Left) projections in the noonmidnight meridian plane (top) and in the equatorial plane (bottom), (right) kinetic energy versus time. The test ions are launched from $65^{\circ}$ latitude with $0.1 \mathrm{eV}$ energy on the noon meridian. Three distinct cross-polar cap potential drops are considered: $1 \mathrm{kV}, 10 \mathrm{kV}$, and $30 \mathrm{kV}$ (coded in grey, blue, and red, respectively).

considered $(\Delta \Phi=1 \mathrm{kV})$, and it can be seen that, in this case, the ion does not flow into the magnetosphere. After a small upward excursion where the ion looses kinetic energy due to gravity, it is turned back and precipitates onto the planet in a manner similar to the parabolic behavior reported by Horwitz (1984) (see Fig. 4 of that paper). The two other trajectories in Fig. 2 exhibit a radically different behavior, with $\mathrm{Na}^{+}$transport in the northern lobe over the polar cap (typically, above $\sim 60^{\circ}$ invariant latitude) and subsequent crossing of the magnetotail current sheet at a radial distance of $\sim 3 \mathrm{R}_{\mathrm{M}}$. For the $\Delta \Phi=10 \mathrm{kV}$ case (coded in blue), the overall circulation occurs within about $10 \mathrm{~min}$ with a two-step ion energization: first up to $\sim 200 \mathrm{eV}$ in the northern lobe, then up to $\sim 5 \mathrm{keV}$ (i.e., about half the total potential drop). For the $\Delta \Phi=30 \mathrm{kV}$ case (coded in red), the $\mathrm{Na}^{+}$transport occurs within $\sim 3$ min, consistent with the typical Dungey cycle time reported by Slavin et al. (2010). In this latter case, the two-step energization is first up to $\sim 1.5 \mathrm{keV}$ in the lobe, then up to $\sim 15 \mathrm{keV}$ in the plasma sheet. A feature of particular interest in Fig. 2 is that, although two distinct cross-polar cap potential drops are considered, the $\mathrm{Na}^{+}$trajectory apexes are quite similar, of the order of $3 \mathrm{R}_{\mathrm{M}}$. Such a result is in sharp contrast with that displayed in Cladis (1986), where the increase in the convection rate, $\Delta \Phi$, leads to a gradual lowering of the ion trajectory apex (see, e.g., the apex evolution obtained from trajectories \#2-4 in Fig. 1 of Cladis, 1986).

To understand this difference between lobe transport at Earth and at Mercury, one must examine the ion energization scheme in detail. For this purpose, the top panels of Fig. 3 show the ion parallel speed versus $\boldsymbol{E} \times \boldsymbol{B}$ drift speed obtained at Earth for $\mathrm{O}^{+}$ions ejected from the dayside cusp ionosphere with different energies. Two distinct cross-polar
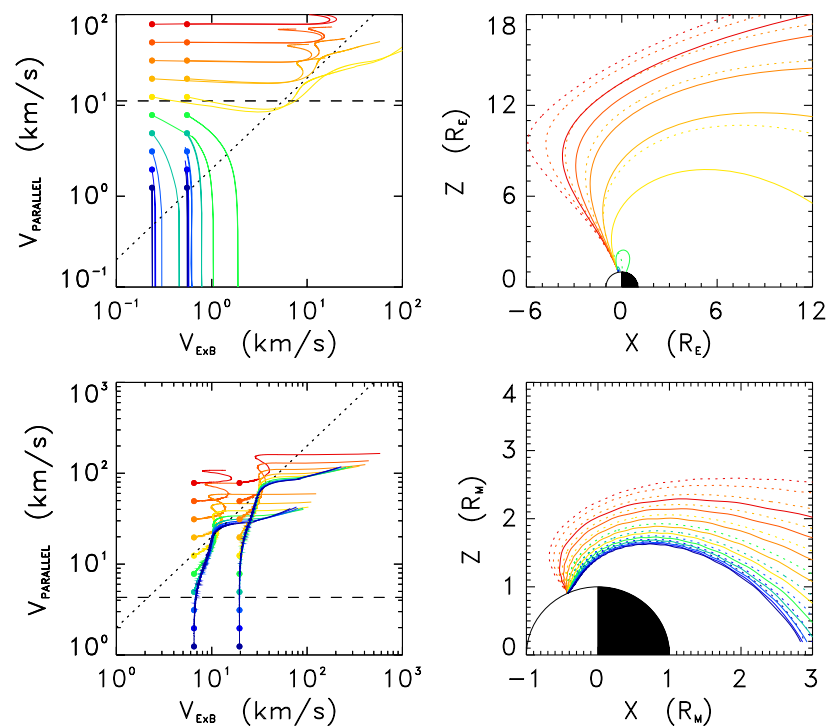

Fig. 3. (Left) Parallel speed of (top) $\mathrm{O}^{+}$ions at Earth and (bottom) $\mathrm{Na}^{+}$ions at Mercury as a function of $\boldsymbol{E} \times \boldsymbol{B}$ drift speed. (Right) Corresponding trajectories in the noon-midnight meridian plane. The test ions are launched into the magnetosphere with different (colorcoded) initial speeds. Two distinct cross-polar cap potential drops $(\Delta \Phi)$ are considered: (top) $60 \mathrm{kV}$ and $120 \mathrm{kV}$, (bottom) $10 \mathrm{kV}$ and $30 \mathrm{kV}$. Closed circles in the left panels show the initial parallel ion speeds and $\boldsymbol{E} \times \boldsymbol{B}$ drift speeds (of $\sim 250 \mathrm{~m} \mathrm{~s}^{-1}$ and $\sim 500 \mathrm{~m} \mathrm{~s}^{-1}$ at Earth, and $\sim 6.5 \mathrm{~km} \mathrm{~s}^{-1}$ and $\sim 20 \mathrm{~km} \mathrm{~s}^{-1}$ at Mercury). Dotted lines in the right panels show the ion trajectories achieved with the lower of the two $\Delta \Phi$.

cap potential drops are also considered here (viz., $60 \mathrm{kV}$ and $120 \mathrm{kV}$ ) that approximately correspond to Case 1 and Case 3 in Fig. 1 of Cladis (1986). Keeping in mind that the escape speed at Earth is $\sim 11 \mathrm{~km} \mathrm{~s}^{-1}$, it can be seen in the top left panel of Fig. 3 that all the ions with initial parallel speeds smaller than this escape speed (horizontal dashed line) are gravitationally bound to the planet and execute parabolic orbits (see Fig. 4 of Horwitz, 1984). Only ions with parallel speeds comparable to or larger than the escape speed travel into the magnetosphere. Most notably, it is apparent in the top left panel of Fig. 3 that, due to the magnetic field topology for Earth with nearly vertical field lines at low altitudes, the ion parallel acceleration after ejection is negligible. It becomes substantial only once the ions reach high altitudes.

This situation is in sharp contrast with that achieved at Mercury, as evidenced by a comparison with the bottom panels of Fig. 3. Here, $\mathrm{Na}^{+}$ions were launched in a similar manner from the planet surface vicinity with different energies and considering two distinct cross-polar cap potential drops $(10 \mathrm{kV}$ and $30 \mathrm{kV})$. In these bottom panels it can be seen that, because of the magnetic field topology that features pronounced field line curvature at low altitudes, ions are immediately and quite significantly accelerated after ejection into the magnetosphere. As a matter of fact, this abrupt 
parallel energization occurs up to a well-defined limit, the parallel speed realized being $\sim 2 V_{E \times B}$ (oblique dotted line in the left panels of Fig. 3), as expected from acceleration by a moving magnetic mirror or Fermi-type energization (see, e.g., Cowley, 1984). This abrupt acceleration at low altitudes plays a decisive role in the overall transport of heavy planetary material, since, regardless of the initial parallel speed (be it well below the particle escape speed), all the ions are systematically launched into the magnetosphere with $\sim 2 V_{E \times B}$ parallel speed and thus readily overcome gravitational trapping unless the convection rate is extremely weak (see, e.g., the trajectory coded in grey in Fig. 2).

\section{Consequences of abrupt curvature-related energization at ejection}

The above Fig. 3 puts forward distinct ion energization schemes for Earth and Mercury, due to the distinct field line topologies of both planets. This explains the differences noted between Fig. 2 and the results of Cladis (1986), with nearly constant trajectory apexes in the former case and apex lowering as $\Delta \Phi$ increases in the latter case (see, e.g., Fig. 1 of Cladis, 1986). The abrupt energization of planetary material obtained at ejection at Mercury has several consequences. First, it is of interest to note that, if all low-energy ions systematically gain a parallel speed of $\sim 2 V_{E \times B}$ immediately after ejection and if no further acceleration mechanism operates during transport over the polar cap, their entry points into the magnetotail do not significantly depend upon cross-polar cap potential drop, since particles $\boldsymbol{E} \times \boldsymbol{B}$ drift in the perpendicular direction over a distance in proportion to that in the parallel direction (the only difference being the time of flight of the particles). This result is clearly apparent from Fig. 2, which shows similar ion paths from the dayside to nightside sectors, but times of flight that differ by a factor of about 3 . In contrast, note that once the ions reach the magnetotail current sheet, their subsequent behavior does depend upon the magnitude of the cross-polar cap potential drop, since it leads to different energies and thus different nonadiabatic regimes (different adiabaticity parameter $\kappa$ defined as the square root of the minimum field line curvature radius-to-maximum Larmor radius ratio).

To further illustrate this result, Fig. 4 shows the altitude and energy of $\mathrm{Na}^{+}$ions at the trajectory apex for two different $\Delta \Phi: 10 \mathrm{kV}$ and $30 \mathrm{kV}$ (coded in grey and black, respectively). The test ions were launched from the planet surface vicinity and from distinct latitudes on the noon meridian (Northern Hemisphere). Also, different current sheet parameters were considered in the modified Luhmann and Friesen (1979) model, using either $L$ or $2 L$, and $B_{\mathrm{T}}$ or $0.5 B_{\mathrm{T}}$. Figure 4 clearly demonstrates that, although the ion energy at apex (right panel) is different by a factor $\sim 3$, as it should be, the apex altitude (left panel) does not significantly vary between $\Delta \Phi=10 \mathrm{kV}$ and $\Delta \Phi=30 \mathrm{kV}$. Thus,
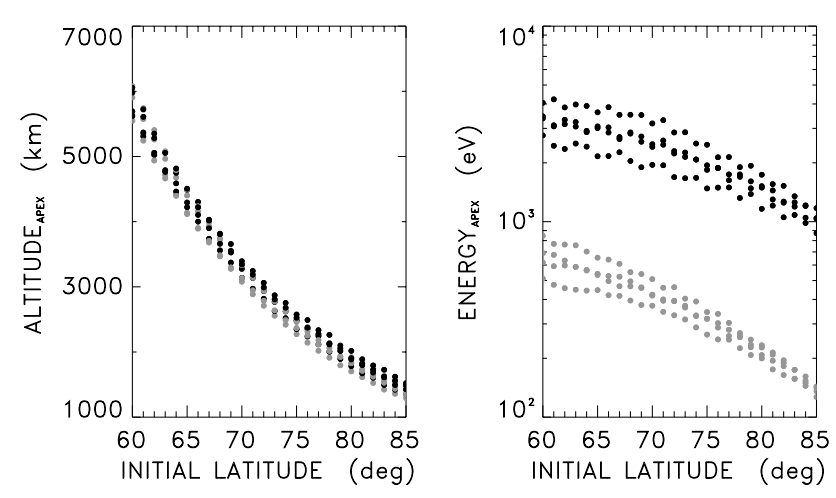

Fig. 4. Altitudes and energies obtained at $\mathrm{Na}^{+}$trajectory apexes as a function of initial latitude. The values obtained for $\Delta \Phi=10 \mathrm{kV}$ and $\Delta \Phi=30 \mathrm{kV}$ are coded in grey and black, respectively. Distinct current sheet parameters are also considered (see text).

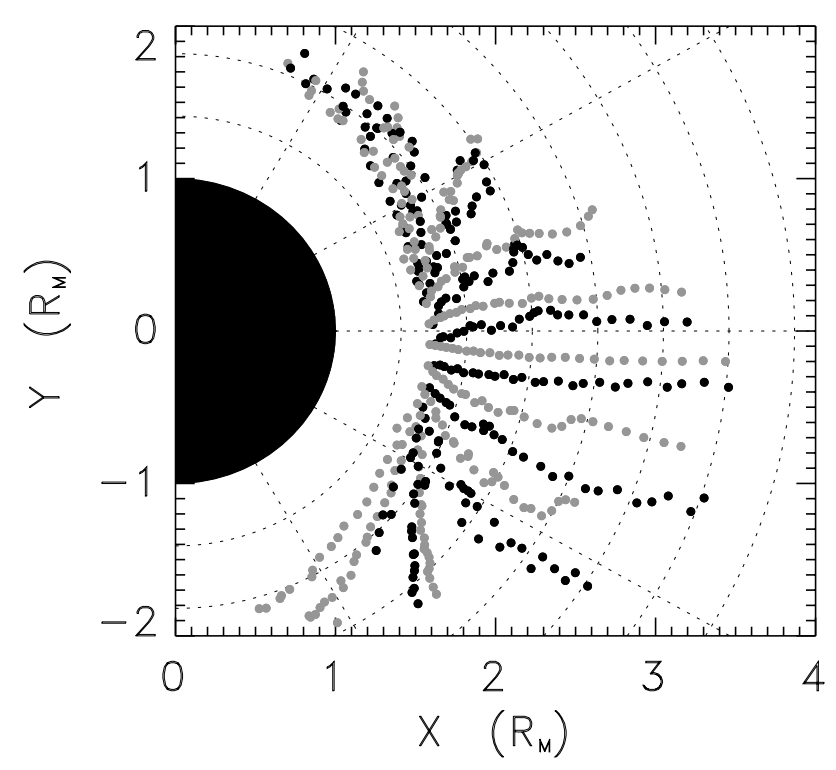

Fig. 5. $\mathrm{Na}^{+}$trajectory apex in the $X-Y$ plane for different initial longitudes and latitudes in the dayside sector. The apexes obtained for $\Delta \Phi=10 \mathrm{kV}$ and $\Delta \Phi=30 \mathrm{kV}$ are coded in grey and black, respectively. Dotted lines show longitudes and altitudes by steps of $1000 \mathrm{~km}$.

regardless of $\Delta \Phi$, the $\mathrm{Na}^{+}$ions are focused into a quite specific region of the nightside sector. Interestingly, it can also be seen in the left panel of Fig. 4 that the trajectory apex of these $\mathrm{Na}^{+}$ions varies from $\sim 6000 \mathrm{~km}$ for an initial latitude of $60^{\circ}$ down to $\sim 1500 \mathrm{~km}$ for an initial latitude of $85^{\circ}$, i.e., a range of altitudes quite comparable to that where enhancements of the observed $\mathrm{Na}^{+}$-group density is noticeable in the MESSENGER data (left panel of Fig. 1).

The location of the $\mathrm{Na}^{+}$trajectory apex on the nightside can be better appreciated in Fig. 5, which displays the results obtained for different initial latitudes and local times in the dayside sector. Grey and black dots in this figure correspond 


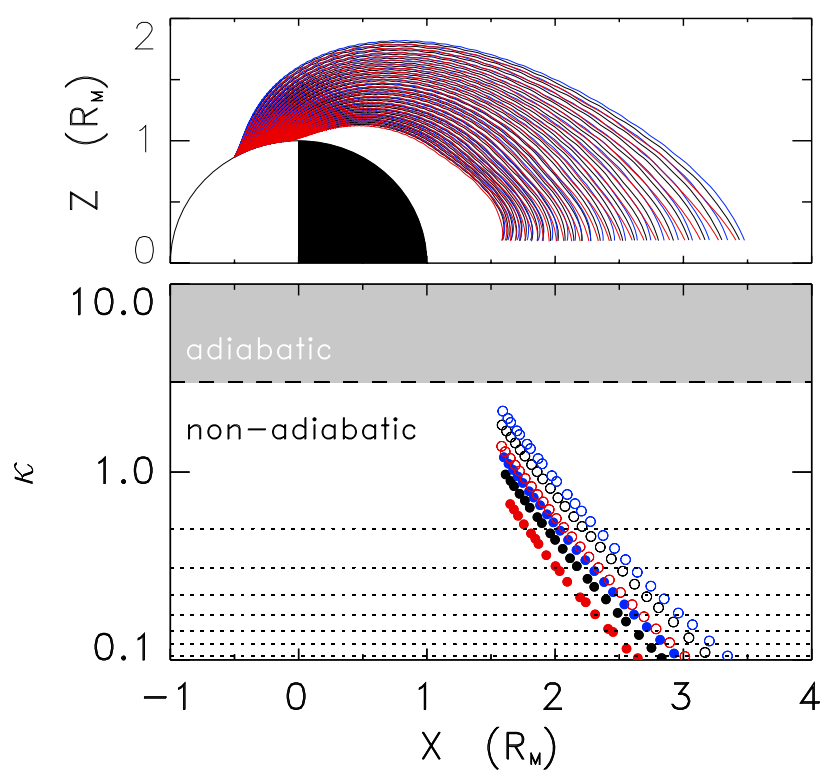

Fig. 6. (Top) Model trajectories (until apex) of $\mathrm{Na}^{+}$(black), $\mathrm{O}^{+}$ (blue) and $\mathrm{Ca}^{+}$(red) in the noon-midnight meridian plane. (Bottom) $\kappa$ parameter of the ions at trajectory apex versus distance $X$. Open and closed circles in the bottom panel show the results obtained for $\Delta \Phi=10 \mathrm{kV}$ and $\Delta \Phi=30 \mathrm{kV}$, respectively. Dotted lines in this panel show the $\kappa$ values for preferentially transient (Speisertype) orbits.

to $\Delta \Phi=10 \mathrm{kV}$ and $\Delta \Phi=30 \mathrm{kV}$, respectively. As mentioned above (see, e.g., Fig. 2), it is apparent from Fig. 5 that the apex altitudinal range does not change significantly with the cross-polar cap potential drop. This altitudinal range maximizes in the pre-midnight sector, where it extends up to $\sim 6000 \mathrm{~km}$. The comparison of Fig. 5 with the FIPS observations in the left panel of Fig. 1 confirms a pronounced similarity between the observed density enhancements and the entry points of planetary $\mathrm{Na}^{+}$into the magnetotail.

Because of the abrupt energization at ejection into the magnetosphere, the dayside-to-nightside paths also depend little upon the ion mass. Regardless of the $m / q$ ratio, the particles rapidly acquire a parallel speed of $\sim 2 V_{E \times B}$ (see Fig. 3), and this allows them to overcome gravity and subsequently travel into the magnetotail over comparable distances. This effect is illustrated in the top panel of Fig. 6, which shows the trajectories (until apex) of $\mathrm{O}^{+}, \mathrm{Na}^{+}$and $\mathrm{Ca}^{+}$ions (coded in blue, black, and red, respectively) launched from different latitudes in the northern dayside sector. This panel clearly demonstrates that, after $\boldsymbol{E} \times \boldsymbol{B}$ convection over the polar cap, the ions reach the magnetotail at similar locations regardless of $m / q$. Here again, this result is in agreement with the MESSENGER FIPS data, which, as noted by Raines et al. (2013), exhibit qualitatively similar features for both $\mathrm{Na}^{+}$and $\mathrm{O}^{+}$(see left and right panels of Fig. 1).
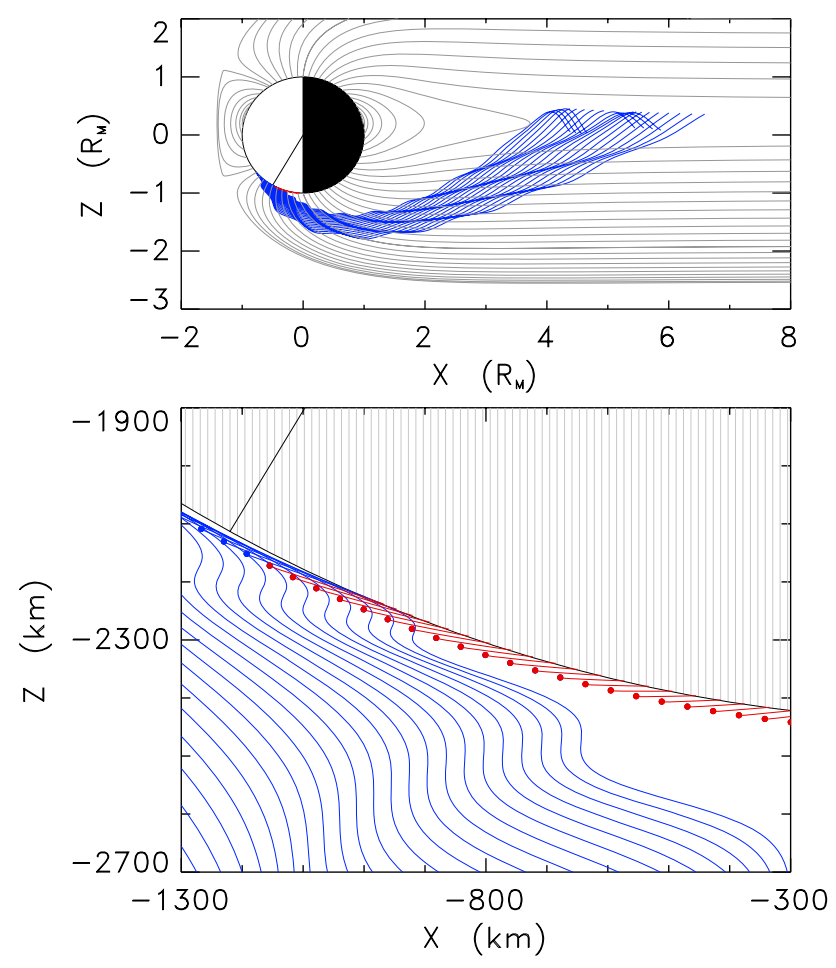

Fig. 7. Model trajectories of $\mathrm{Na}^{+}$ions ejected from the southern dayside sector. The bottom panel shows an enlarged view of the $\mathrm{Na}^{+}$paths at low altitudes. Ions that do not gain access to the magnetotail are coded in red. The solid line in both panels depicts the $-60^{\circ}$ latitude.

Still, subsequent interaction of the ions with the magnetotail current sheet will differ owing to the different ion Larmor radii (different adiabaticity parameter $\kappa$ ). This is summarized in the bottom panel of Fig. 6, which shows the $\kappa$ profiles of $\mathrm{O}^{+}, \mathrm{Na}^{+}$and $\mathrm{Ca}^{+}$at the trajectory apex for two different values of $\Delta \Phi(10 \mathrm{kV}$ and $30 \mathrm{kV}$, coded with open and closed circles, respectively). This panel provides insights into the upper $\kappa$ limit realized as a function of radial distance in the magnetotail, since these ions were ejected at very low energies (ions with larger energies will exhibit smaller $\kappa$ values upon interaction with the current sheet). It can be seen in the bottom panel of Fig. 6 that $\kappa$ is somewhat larger for $\mathrm{O}^{+}$than for $\mathrm{Ca}^{+}$, as expected from smaller Larmor radii, and that $\kappa$ is in all cases smaller than 3 (note also the smaller $\kappa$ values achieved when $\Delta \Phi$ increases due to the larger velocity realized). Keeping in mind that $\kappa \approx 3$ delineates adiabatic (magnetic moment conserving) and nonadiabatic regimes, Fig. 6 confirms the suggestion made by Raines et al. (2013) that most of the Hermean magnetotail is nonadiabatic for heavy material of planetary origin. In this nonadiabatic regime, particles may exhibit either chaotic behaviors (particularly at $\kappa \approx 1$ ) or transient (Speiser-type) orbits in limited intervals of $\kappa<1$ (e.g., Büchner and Zelenyi, 1989). 
On the other hand, it was mentioned above that the modified Luhmann and Friesen (1979) model used here takes into account the northward offset of the magnetic field reported by Anderson et al. (2011). Since this offset is responsible for an asymmetry between the Northern and Southern hemispheres, it is of interest to examine the behavior of ions ejected from the planet surface vicinity in the Southern Hemisphere and to compare it with the above results for ions originating in the Northern Hemisphere. This is illustrated in Fig. 7, which shows the trajectories obtained for $\mathrm{Na}^{+}$ions launched with $0.1 \mathrm{eV}$ energy from different latitudes along the noon meridian in the Southern Hemisphere. Note that the offset dipole leads to a weaker magnetic field in the southern polar cap as compared to the northern one; hence, larger Larmor radii and a more pronounced "wavy" character of the ion paths in Fig. 7. It is apparent from the top panel of Fig. 7 that, in a like manner to the Northern Hemisphere, planetary ions can gain access to the magnetotail over the polar cap, although their initial speed is well below the escape speed. As examined above (Fig. 3), this follows from abrupt curvature-related energization after ejection into the magnetosphere. Also, it was shown in Fig. 3 that the particle speed increases in proportion to the $\boldsymbol{E} \times \boldsymbol{B}$ drift speed, so that the particle path depends little upon the cross-polar cap potential drop, and the same result holds for ions originating in the Southern Hemisphere, with little variations in the computed paths for $\Delta \Phi=10 \mathrm{kV}$ and $\Delta \Phi=30 \mathrm{kV}$ (not shown).

Still, because of the different field line topology in the Southern Hemisphere as compared to the Northern, it can be seen in the top panel of Fig. 7 that $\mathrm{Na}^{+}$ions are here transported further out into the magnetotail, typically beyond $4 \mathrm{R}_{\mathrm{M}}$. At such distances, ions are essentially lost into the dusk magnetopause after intercepting the magnetotail current sheet, so that they do not travel back toward the planet and do not contribute to the plasma population of the inner magnetosphere. Moreover, the field line topology at low altitudes in the Southern Hemisphere is such that a number of ions originating at high latitudes are rapidly turned back toward the planet. Indeed, it is apparent from the trajectories coded in red in the bottom panel of Fig. 7 that all the $\mathrm{Na}^{+}$ions initialized above $\sim 60^{\circ}$ latitude in the Southern Hemisphere do not escape into the magnetosphere and are immediately convected back to the planet surface. Thus, in contrast to ions originating in the Northern Hemisphere that experience bulk focusing into a limited range of altitudes in the inner Hermean magnetotail, ions originating from the Southern Hemisphere may not contribute significantly to the plasma populations of this region of space.

\section{Conclusions}

Because of the small spatial scales of Mercury's environment, heavy ions that are released at very low energies (a few tenths of eVs) near the dayside planet surface due to ionization of exospheric neutrals, are rapidly accelerated up to $\sim 2 V_{E \times B}$. This abrupt parallel acceleration which is analogous to that due to a moving magnetic mirror is specific to Mercury's environment and strongly contrasts with the ion energization scheme obtained at Earth. This mechanism is of importance for the supply of plasma to the inner Hermean magnetotail, since it not only allows the heavy material of planetary origin to overcome gravity, but also leads to bulk focusing of this material into a specific domain of the magnetotail. Test ion trajectory computations suggest that this domain lies in the $\sim 1000-6000 \mathrm{~km}$ altitudinal range in the central magnetotail, which is in agreement with the plasma observations from the FIPS instrument on board MESSENGER that exhibit prominent density enhancements in this very region of space. Since a parallel speed of $\sim 2 V_{E \times B}$ is realized regardless of the ion mass-to-charge ratio, all ions are $\boldsymbol{E} \times \boldsymbol{B}$ convected into a similar region of the magnetotail, with little dependence upon convection rate, a feature that is also in agreement with the FIPS observations that reveal qualitatively similar patterns for both the $\mathrm{Na}^{+}$group and the $\mathrm{O}^{+}$group. Due to the northward offset of the planetary magnetic field, an asymmetry is found between ion transport from the Northern and Southern hemispheres in the dayside sector, ions originating from the Northern Hemisphere preferentially contributing to the populations of the inner Hermean magnetotail.

Acknowledgements. The author thanks J. M. Raines for providing him with the MESSENGER FIPS data for Fig. 1.

Topical Editor I. Daglis thanks J. A. Slavin and J. M. Raines for their help in evaluating this paper.

\section{References}

Anderson, B. J., Johnson, C. L., Korth, H., Purucker, M. E., Winslow, R. M., Slavin, J. A., Solomon, S. C., McNutt Jr., R. L., Raines, J. M., and Zurbuchen, T. H.: The global magnetic field of Mercury from MESSENGER orbital observations, Science, 333, 1859-1862, 2011.

Büchner, J. and Zelenyi, L. M.: Regular and chaotic charged particle motion in magnetotaillike field reversals : 1 . Basic theory of trapped motion, J. Geophys. Res., 94, 11821-11842, 1989.

Cladis, J. B.: Parallel acceleration and transport of ions from polar ionosphere to plasma sheet, Geophys. Res. Lett., 13, 893-896, 1986.

Cowley, S. W. H.: The distant geomagnetic tail in theory and observation, AGU Monograph on "Magnetic reconnection in space and laboratory plasmas", Vol. 30, p. 228, 1984.

Delcourt, D. C., Sauvaud, J.-A., and Perdersen, A.: Dynamics of single-particle orbits during substorm expansion phase, J. Geophys. Res., 95, 20853-20865, 1990.

Delcourt, D. C., Moore, T. E., Orsini, S., Millilo, A., and Sauvaud, J.-A.: Centrifugal acceleration of ions near Mercury, Geophys. Res. Lett., 29, 32.1-32.4, doi:10.1029/2001GL013829, 2002.

Horwitz, J. L.: Features of ion trajectories in the polar magnetosphere, Geophys. Res. Lett., 11, 1111-1114, 1984. 
Hunten, D. M., Morgan, T. H., and Shemansky, D. E.: The Mercury atmosphere, Mercury, The University of Arizona Press, 562 pp., 1988.

Leblanc, F. and Johnson, R. E.: Mercury exosphere I. Global circulation model of its sodium component, Icarus, 209, 280-300, 2010.

Luhmann, J. G. and Friesen, L. M.: A simple model of the magnetosphere, J. Geophys. Res., 84, 4405-4408, 1979.

Northrop, T. G.: The Adiabatic Motion of Charged Particles, Wiley Interscience, New York, 1963.

Raines, J. M., Gershman, D. J., Zurbuchen, T. H., Sarantos, M., Slavin, J. A., Gilbert, J. A., Korth, H., Anderson, B. J., Gloeckler, G., Krimigis, S. M., Baker, D. N., McNutt Jr., R. L., and Solomon, S. C.: Distribution and compositional variations of plasma ions in Mercury's space environment: The first three Mercury years of MESSENGER observations, J. Geophys. Res., 118, 1604-1619, doi:10.1029/2012JA018073, 2013.

Slavin, J. A., Anderson, B. J., Baker, D. N., Benna, M., Boardsen, S. A., Gloeckler, G., Gold, R. E., Ho, G. C., Korth, H., Krimigis, S. M., McNutt Jr., R. L., Nittler, L. R., Raines, J. M., Sarantos, M., Schriver, D., Solomon, S. C., Starr, R. D., Trávníček, P. M., and Zurbuchen, T. H.: MESSENGER observations of extreme loading and unloading of Mercury's magnetic tail, Science, 329, 665-668, 2010.
Volland, H.: A model of the magnetospheric convection electric field, J. Geophys. Res., 83, 2695-2699, 1978.

Zurbuchen, T. H., Raines, J. M., Gloeckler, G., Krimigis, S. M., Slavin, J. A., Koehn, P. L., Killen, R. M., Sprague, A. L., McNutt Jr., R. L., and Solomon, S. C.: MESSENGER observations of the composition of Mercury's ionized exosphere and plasma environment, Science, 321, 90-92, 2008.

Zurbuchen, T. H., Raines, J. M., Slavin, J. A., Gershman, D. J., Gilbert, J. A., Gloeckler, G., Anderson, B. J., Baker, D. N., Korth, H., Krimigis, S. M., Sarantos, M., Schriver, D., McNutt Jr., R. L., and Solomon, S. C.: MESSENGER observations of the spatial distribution of planetary ions near Mercury, Science, 333, 1862-1865, 2011. 